\title{
A DIVISIBILITY PROPERTY OF THE BERNOULLI POLYNOMIALS
}

\author{
L. CARLITZ
}

1. Put

$$
\frac{x e^{x u}}{e^{x}-1}=\sum_{m=0}^{\infty} B_{m}(u) \frac{x^{m}}{m !}, \quad B_{m}=B_{m}(0) .
$$

The writer has shown $[1, \S 4]$ that if $(p-1) p^{r} \mid m, m>0$, then

$$
B_{m}+\frac{1}{p}-1 \equiv 0\left(\bmod p^{r}\right)
$$

indeed if $m=t(p-1) p^{r}$, then

$$
\sigma_{m}=\frac{1}{p^{r}}\left(B_{m}+\frac{1}{p}-1\right) \equiv t w_{p}(\bmod p),
$$

where $w_{p}=((p-1) !+1) / p$. (For the case $r=0$, see $[2$, p. 354].) It was stated that

$$
\sigma_{m} \equiv p^{1-h} \sum_{a=1, p \chi_{a}}^{p h} q(a)\left(\bmod p^{2 h}\right)
$$

where $h=[(r+2) / 3]$ and $q(a)=\left(a^{(p-1)} p^{r}-1\right) / p^{r+1}$.

In the present note we first extend these results to $B_{m}(u)$, where the rational number $u$ is integral $(\bmod p)$. Secondly we derive the corresponding divisibility property for $B_{m}^{(k)}(u)$ defined by [3, chap. 6]

$$
\left(\frac{x}{e^{x}-1}\right)^{k} e^{x u}=\sum_{m=0}^{\infty} B_{m}^{(k)}(u), \quad B_{m}^{(k)}=B_{m}^{(k)}(0),
$$

where $k$ is restricted to the range $1 \leqq k \leqq p-1$.

2. We recall that

$$
\frac{1}{m+1}\left(B_{m+1}(u+t)-B_{m+1}(u)\right)=\sum_{s=0}^{t-1}(u+s)^{m}
$$

also

$$
B_{m}(u+t)=\sum_{s=0}^{m}\left(\begin{array}{c}
m \\
s
\end{array}\right) t^{s} B_{m-8}(u) .
$$

Presented to the Society, February 23, 1952; received by the editors November 21, 1951. 
Now for $p \nmid u+s$, put

$$
(u+s)^{(p-1) p r}=1+p^{r+1} q(u+s),
$$

from which it follows that

$$
(u+s)^{m} \equiv 1+t p^{r+1} q(u+s)\left(\bmod p^{2 r+2}\right) \quad\left(m=t(p-1) p^{r}\right) .
$$

If we put

$$
S_{m}\left(p^{h}\right)=\sum_{s=0}^{p h-1}(u+s)^{m}, \quad S_{m}^{\prime}\left(p^{h}\right)=\sum_{s=0, p h_{u+s}}^{p h-1}(u+s)^{m},
$$

where $h \geqq 1$, it is evident that

$$
S_{m}\left(p^{h}\right) \equiv S_{m}^{\prime}\left(p^{h}\right)\left(\bmod p^{2 r+2}\right),
$$

since $m \geqq(p-1) p^{r} \geqq 2(r+1)$. In the next place it follows from (2.3) that

$$
S_{m}^{\prime}\left(p^{h}\right) \equiv p^{h}-p^{h-1}+t p^{r+1} R\left(p^{h}\right)\left(\bmod p^{2 r+2}\right)
$$

where

$$
R\left(p^{h}\right)=\sum_{s=0, p \ell_{u+s}^{p h-1}}^{p^{n}} q(u+s) .
$$

By (2.1) and (2.2) we see that

$$
\begin{aligned}
S_{m}\left(p^{h}\right) & =\frac{1}{m+1} \sum_{s=1}^{m+1}\left(\begin{array}{c}
m+1 \\
s
\end{array}\right) p^{h s} B_{m+1-s}(u) \\
& =\sum_{s=0}^{m} \frac{1}{s+1}\left(\begin{array}{c}
m \\
s
\end{array}\right) p^{h(s+1)} B_{m-s}(u) .
\end{aligned}
$$

Now let $p \geqq 3$; then it is easily verified that for $s \geqq 1$ each term in the extreme right member of (2.6) is divisible by at least $p^{r+2 h}$. Hence by (2.4), (2.5), (2.6) we have

(2.7) $\quad p^{h} B_{m}(u)+p^{h-1}-p^{h} \equiv t p^{r+1} R\left(p^{h}\right)\left(\bmod p^{2 r+2}, p^{r+2 h}\right)$.

In particular for $h=1,(2.7)$ becomes

$$
p B_{m}(u)+1-p \equiv t p^{r+1} R(p)\left(\bmod p^{r+2}\right)
$$

which shows that

$$
\sigma_{m}(u)=\frac{1}{p^{r}}\left(B_{m}(u)+\frac{1}{p}-1\right)
$$


is integral $(\bmod p)$ and indeed

$$
\sigma_{m}(u) \equiv t R(p)(\bmod p) .
$$

It is easily seen that, for $u=0,(2.9)$ reduces to (1.3).

To get a stronger congruence we take $2 h \leqq r+2$. Clearly (2.7) implies

$$
\sigma_{m}(u) \equiv t p^{1-h} R\left(p^{h}\right)\left(\bmod p^{h}\right) \quad(1 \leqq h \leqq r / 2+1) .
$$

In particular when $h=[r / 2+1]$, we have the largest modulus.

We now state:

Theorem 1. Let $p \geqq 3, m=t(p-1) p^{r}$, then $\sigma_{m}(u)$ as defined by $(2.8)$ is integral $(\bmod p)$. Moreover $\sigma_{m}(u)$ satisfies the congruences (2.9) and (2.10).

For $u=0,(2.10)$ is not quite as sharp as (1.4). Indeed to prove (1.4) we take $u=0$ in (2.6) and assume $p>3$. Then since $B_{m-1}=0$ we can show that (2.6) implies

$$
S_{m}\left(p^{h}\right) \equiv p^{h} B_{m}\left(\bmod p^{r+3 h}\right)
$$$$
(u=0) \text {; }
$$

the rest of the argument is as before except that we take $3 h \leqq r+2$. Thus (1.4) is proved.

We remark that if in place of (2.3) we use

$$
(u+s)^{m}=1+p^{r+1} Q(u+s)
$$

so that $Q$ is integral $(\bmod p)$, and replace $(2.4)$ by the stronger congruence

$$
S_{m}\left(p^{h}\right) \equiv S_{m}^{\prime}\left(p^{h}\right)\left(\bmod p^{m}\right),
$$

then (2.7) becomes

$$
p^{h} B_{m}(u)+p^{h-1}-p^{h} \equiv p^{r+1} R^{*}\left(p^{h}\right)\left(\bmod p^{m}, p^{r+2 h}\right),
$$

where now

$$
R^{*}\left(p^{h}\right)=\sum_{s=0, p h_{u+s}}^{p^{h-1}} Q(u+s) .
$$

Hence

$$
\sigma_{m}(u) \equiv p^{1-h} R^{*}\left(p^{h}\right)\left(\bmod p^{h}\right),
$$

provided $r+2 h \leqq m$.

Similarly in the case $u=0$, we find that

$$
\sigma_{m} \equiv p^{1-h} R^{*}\left(p^{h}\right)\left(\bmod p^{h}\right) \quad(p>3),
$$


provided $r+3 h \leqq m$.

3. We shall require the following formula $[3$, p. $148,(87)]$ :

$$
B^{(k)}(u)=k\left(\begin{array}{c}
m \\
k
\end{array}\right) \sum_{s=0}^{k-1}(-1)^{k-1-s}\left(\begin{array}{c}
k-1 \\
s
\end{array}\right) \frac{B_{m-8}(u)}{m-s} E_{s}^{(k)}(u),
$$

where $B_{m}^{(k)}(u)$ is defined by (1.5). We suppose $m \equiv s_{0}(\bmod p-1)$, $0 \leqq s_{0} \leqq k-1, k \leqq p-1$; also $p^{r} \mid m-s_{0}$. Now for $s \neq s_{0}$, both $B_{m-8}(u)$ and $B_{s}^{(k)}(u)$ are integral $(\bmod p)$. Hence $(3.1)$ implies

$$
B_{m}^{(k)}(u) \equiv(-1)^{k-1-s_{0}}\left(\begin{array}{c}
m \\
s_{0}
\end{array}\right)\left(\begin{array}{c}
m-s_{0}-1 \\
k-s_{0}-1
\end{array}\right) B_{m-s_{0}}(u) B_{s_{0}}^{(k)}(u)\left(\bmod p^{r}\right),
$$

so that by Theorem 1 ,

$$
\begin{aligned}
\sigma_{m}^{(k)}(u)=\frac{1}{p^{r}} & \left\{B_{m}^{(k)}(u)\right. \\
& \left.+(-1)^{k-s_{0}}\left(1-\frac{1}{p}\right)\left(\begin{array}{c}
m \\
s_{0}
\end{array}\right)\left(\begin{array}{c}
m-s_{0}-1 \\
k-s_{0}-1
\end{array}\right) B_{s_{0}}^{(k)}(u)\right\}
\end{aligned}
$$

is integral $(\bmod p)$. We state:

THEOREM 2. Let $p \geqq 3,1 \leqq k \leqq p-1 ; m \equiv s_{0}(\bmod p-1), 0 \leqq s_{0} \leqq k-1$; $p^{r} \mid m-s_{0}$; then $\sigma_{m}^{(k)}(u)$ as defined by (3.2) is integral $(\bmod p)$. In particular if $(p-1) p^{r} \mid m$ then

$$
\frac{1}{p^{r}}\left\{B_{m}^{(k)}(u)+(-1)^{k}\left(1-\frac{1}{p}\right)\left(\begin{array}{c}
m-1 \\
k-1
\end{array}\right)\right\}
$$

is integral $(\bmod p)$.

Theorem 2 can be extended to larger values of $k$ but the results are complicated. We remark that

$$
B_{s}^{(k)}(u)=\frac{s !}{(k-1) !}\left(\frac{d}{d u}\right)^{k-1-s}(u-1)(u-2) \cdots(u-k+1)
$$

for $k>s$.

\section{REFERENCES}

1. L. Carlitz, Some congruences for the Bernoulli numbers, Amer. J. Math.

2. Emma Lehmer, On congruences involving Bernoulli numbers and the quotients of Fermat and Wilson, Ann. of Math. (2) vol. 39 (1938) pp. 350-360.

3. N. E. Nörlund, Vorlesungen ïber Differenzenrechnung, Berlin, 1924.

DUKE UNIVERSITY 